\title{
ANÁLISIS BIBLIOMÉTRICO DE LA PRODUCCIÓN CIENTÍFICA DE MÉXICO EN CIENCIAS AGRÍCOLAS DURANTE EL PERIODO 1983-2002
}

\author{
BIBLIOMETRIC ANALYSIS OF THE MEXICAN SCIENTIFIC PRODUCTION IN AGRICULTURAL \\ SCIENCES DURING THE YEARS 1983-2002
}

\author{
Ángel Bravo-Vinaja ${ }^{1}$ y Elías Sanz-Casado ${ }^{2}$
}

\begin{abstract}
${ }^{1}$ Centro de Documentación y Biblioteca. Carretera México-Texcoco. Km. 36.5. 56230, Montecillo, Texcoco, Estado de México. Tel. (55) 5804 5931, (595) 952 0231, (595) $9520231^{2}$ Departamento de Biblioteconomía y Documentación, Universidad Carlos III de Madrid. C/ Madrid, 126 - 28903, Getafe, Madrid, España.
\end{abstract}

*Autor para correspondencia (abravo@colpos.mx)

\section{RESUMEN}

En este trabajo se caracterizó mediante indicadores bibliométricos, la actividad científica de las instituciones mexicanas en ciencias agrícolas, a partir de los artículos arbitrados y contenidos en las bases de datos: Agricola, Agris, Cab Abstracts, Tropag \& Rural, Science Citation Index (SCI) y Social Science Citation Index (SSCI). En las primeras cuatro bases de datos solamente se dispuso de la dirección laboral del autor para correspondencia, que generalmente es el primer autor. Los indicadores permitieron caracterizar la evolución de los artículos publicados en revistas, la producción por entidades federativas de México, sectores institucionales e instituciones individuales, los idiomas en que publican, la colaboración entre autores y los índices de coautoría. La producción científica durante el periodo de estudio ascendió a 15736 artículos. El Distrito Federal y el Estado de México publicaron más de la mitad de los artículos, lo cual muestra una alta concentración de la investigación en estos dos estados. Los sectores institucionales que mostraron mayor actividad investigadora fueron las universidades públicas y los institutos o centros de investigación. Los idiomas en que se publicaron más artículos de revistas fueron el inglés y el español. La tasa media de artículos firmados en coautoría fue de $87.62 \%$; el índice de coautoría aumentó de 2.47 autores por artículo en 1983 a 4.08 en 2002.

Palabras clave: México, ciencias agrícolas, análisis biliométrico, bases de datos, producción científica.

\section{SUMMARY}

In this research the Mexican scientific production in agricultural sciences is characterized, by means of bibliometric indicators, from the production included in several databases: Agricola, Agris, Cab Abstracts, Tropag \& Rural, Science Citation Index (SCI) and Social Science Citation Index (SSCI). In the first four databases, only the address of the corresponding author was available, who usually is the first author. The indicators used here allow to characterize the evolution of articles published in journals, the national contribution by states and by institutions and sectors, the languages in which the articles are published, the collaboration between authors and the coauthorship index. The scientific production during the evaluated period ascended to 15736 journal articles. Two states, the Federal District and the State of México, published more than half of articles thus showing a high concentration of the national scientific research. The institutions that published more articles were public universities and institutes or research centers. The languages in which more journal articles were published were English and Spanish. The average rate of articles signed in coauthorship was of $87.62 \%$. The coauthorship index increased from 2.47 authors per article in 1983 to 4.08 in 2002.

Index words: México, agricultural sciences, bibliometric analysis, databases, scientific literature.

\section{INTRODUCCIÓN}

El desarrollo científico y tecnológico en México ha sido condicionado por factores políticos, sociales y económicos que han afectado al país a lo largo de su historia, y es a partir de la mitad del siglo XX cuando se inició la profesionalización de la investigación científica en el sector educativo, al crearse plazas de profesores e investigadores de tiempo completo, principalmente con la instauración del Centro de Investigación y de Estudios Avanzados (CINVESTAV) del Instituto Politécnico Nacional (IPN) (Aréchiga, 1995) y profesores-investigadores en el Colegio de Postgraduados. Durante la década de los setenta se impulsó el sistema de instituciones de educación superior del país, y la inversión en ciencia y tecnología aumentó de manera sostenida. En 1971 se fundó el Consejo Nacional de Ciencia y Tecnología (CONACYT), y en esa década se crearon también nuevos centros de investigación dependientes del Consejo, hoy denominado Sistema CONACYT (Aréchiga, 1995).

La creación en 1984 del Sistema Nacional de Investigadores (SNI) fue una estrategia para paliar el deterioro del salario de los investigadores y evitar una inminente deserción y desmantelamiento de los cuadros científicos. 
En México no existe un sistema formal que coordine las actividades de las diferentes instituciones de investigación agropecuaria, pero hay agentes públicos y privados que interactúan entre sí y que efectúan investigación y extensión agropecuaria (Ekboir et al., 2003). La Ley para el Fomento de la Investigación Científica y Tecnológica (27 de abril de 1999), creó la figura jurídica de los Centros Públicos de Investigación a los que se otorga mayor autonomía técnica, operativa y administrativa, además de facilitar el acceso a recursos financieros para sostener e incrementar sus programas de investigación.

La ciencia constituye una actividad de importancia en el contexto social, que genera el conocimiento cierto, riguroso y sistemático, basado en la recopilación de datos empíricos guiados por un sistema racional y obtenidos con una metodología objetiva, cuyas implicaciones económicas son trascendentes (González, 1997). Por ello la evaluación de la actividad científica es imprescindible cuando su financiamiento se realiza con fondos públicos, la mayoría de las veces escasos en los países en vías de desarrollo; tal interés ha conducido a la búsqueda de métodos objetivos para evaluar la actividad científica que complementen la evaluación realizada por pares o colegas. Un método objetivo es utilizar indicadores bibliométricos de producción y colaboración, que se basan en el análisis de las publicaciones científicas y tecnológicas, en una institución, país o región del mundo (Spinak, 1996).

En México se han hecho diversos estudios sobre la producción científica, entendida ésta como la publicación de documentos sometidos a un proceso de arbitraje y revisión editorial. Particularmente en ciencias agrícolas, la mayoría de estudios se han enfocado al área de ciencia animal y veterinaria (Anta et al., 1989; Galina et al., 2000; D'alessandro et al., 2000; Lomnitz y Mayer, 1994; Mirande et al., 1987; Russell et al., 1987; Russell y Galina, 1987, 1988). Otras áreas que se han estudiado son: recursos naturales (Gaillard et al., 2001), ciencia y tecnología de alimentos (Alfaraz y Calviño, 2004), y las ciencias agrícolas (Arenas et al., 2004; Licea et al., 2003; Saavedra et al., 2002).

En este trabajo se estudia la producción científica en México en ciencias agrícolas, en el periodo 1983-2002, incluida en cuatro bases de datos especializadas en agricultura y en dos bases de datos generales, con los objetivos de caracterizar e identificar las tendencias en la producción científica en los veinte años analizados, las entidades federativas más productivas y los tipos de institución involucrados, las instituciones más productivas, los idiomas de publicación, las revistas en las que se publicó $50 \%$ de los artículos, la colaboración científica entre autores y el índice de coautoría.

\section{MATERIALES Y MÉTODOS}

Se utilizó la información de los artículos publicados durante el periodo 1983-2002 en las siguientes bases de datos bibliográficas publicadas en disco compacto: Agricola, Agris, Cab Abstracts, Science Citation Index (SCI), Social Sciences Citation Index (SSCI) y Tropag \& Rural. Las bases de datos "agrícolas" tienen la limitante de que solamente incluyen los datos de filiación del autor responsable de la correspondencia, que generalmente es el primer autor. Además, las bases de datos SCI y SSCI sólo incluyen los datos de revistas que ISI Thomson certifica como de relevancia, por lo que la mayoría de las revistas editadas en español quedan fuera de esta base de datos. El criterio de búsqueda fue que el campo de filiación o dirección de los autores incluyera alguna institución asentada en México. Los registros recuperados se importaron a una base de datos diseñada ex profeso en el manejador de referencias bibliográficas ProCite ${ }^{\mathrm{TM}}$, a través de la utilería BiblioLink $^{\mathrm{TM}}$. Una vez que los registros duplicados se eliminaron, el total de registros fue 15736 , de los cuales 1185 (7.53 \%) se obtuvieron de Agricola, 2487 (15.74 \%) de Agris, 9456 de CAB Abstracts (60.09 \%), 168 (1.06\%) de Tropag \& Rural, 2449 (15.50\%) de SCI, y $10(0.64 \%)$ de SSCI. Los cálculos y análisis estadísticos (correlación, $\mathrm{r}$; índice de determinación, $\mathrm{R}^{2}$; pendiente; $\mathrm{y}$ media) se hicieron en Excel para Windows.

Los indicadores usados para el análisis bibliométrico fueron la producción de artículos científicos por área geográfica local y por tipo de institución; para el análisis se agruparon en siete categorías o tipos de instituciones: a) Universidades públicas y privadas, que incluye a los institutos de investigación incorporados a estas universidades; b) Institutos o Centros de investigación, correspondientes a las entidades públicas o privadas dedicadas principalmente a la investigación, sean o no centros públicos de investigación; c) Organismos internacionales con centros o unidades de investigación en territorio mexicano; d) Hospitales, que incorpora a unidades hospitalarias e institutos que realizan investigación en salud; e) Gobierno, que incluye a las instituciones gubernamentales que dependen directamente del estado, como secretarías de estado e instituciones públicas que no tienen independencia en su gestión o administración; f) Industrias o empresas con actividad investigadora; y finalmente g) "Otro tipo" de instituciones, que generalmente engloba a asociaciones sin fines de lucro, como fundaciones y a autores que firmaron como particulares. También se utilizaron como indicadores la lengua de publicación y la colaboración entre autores. Esta colaboración se determinó a través del índice de coautoría, el cual permite determinar el grado de cooperación científica entre autores (Sancho, 1990), y se 
calculó como el cociente del número de autores entre el número de trabajos publicados.

\section{RESULTADOS Y DISCUSIÓN}

\section{Producción científica}

La producción científica anual durante el periodo de estudio se distribuyó con una tendencia de crecimiento lineal, aunque con algunas fluctuaciones a la baja en los años 1989 y 2001 (Figura 1). La media de producción anual fue de 787 artículos. La producción en la segunda mitad del periodo superó a la media global que fue de 571 artículos por año, puesto que en los años 1993-2002 fueron 1002 artículos. Si bien estos datos permiten conocer la actividad científica mexicana en ciencias agrícolas, hay que considerar que las bases de datos seleccionan los documentos de acuerdo con sus propias políticas (Russell et $a l .$, 1990). Además según Nasir et al. (1994), debido a la falta de un control bibliográfico adecuado hay una cantidad importante de literatura que no llega a los servicios de indexación.

\section{Producción científica por entidad federativa}

En conjunto, más de $50 \%$ de la producción científica se concentró en el Distrito Federal (DF) y el Estado de México (30.77\% y $22.71 \%$, respectivamente), que es la zona donde tienen su sede muchos centros de investigación en ciencias agrícolas. Entre los estados con instituciones cuyos investigadores publicaron menos artículos en revistas científicas se encuentran: Quintana Roo, Guerre- ro, Tlaxcala, Campeche e Hidalgo (Cuadro 1). La alta tasa de producción científica en ciencias agrícolas en el Estado de México también ha sido observada por otros autores, según Licea et al. (2003), en este estado se produjeron más trabajos en el país firmados por investigadoras $(59.31 \%)$, seguido del DF, Sonora, Chiapas y Puebla; estos últimos datos deben tomarse con reserva, ya que sólo se analizó a investigadoras del área agrícola pertenecientes al SNI. En el tema de la reproducción bovina en el trópico (Anta et al., 1989), el estado con más estudios fue Veracruz, seguido de Yucatán, Tabasco y Nayarit, todos ellos con clima tropical, lo que muestra que estudios sobre temas de interés local se desarrollan en lugares específicos.

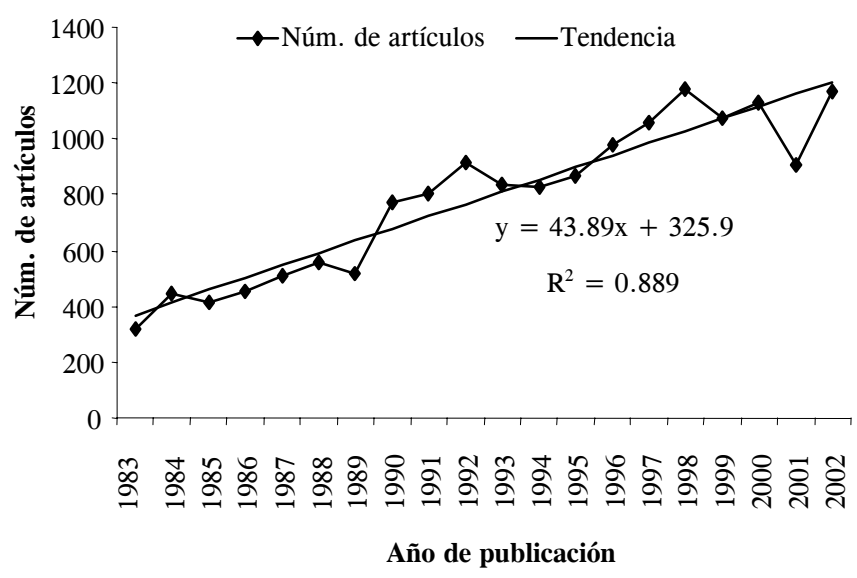

Figura 1. Producción anual de artículos de revistas en ciencias agrícolas en México (1983-2002).

Cuadro 1. Número y tasa de participación en la producción de artículos de revistas científicas en ciencias agrícolas por estados geográficos de México (1983-2002).

\begin{tabular}{|c|c|c|c|c|c|}
\hline Estado & Núm. de artículos & $\%$ & Estado & Núm. de artículos & $\%$ \\
\hline Distrito Federal & 4842 & 30.77 & Chihuahua & 173 & 1.10 \\
\hline Estado de México & 3573 & 22.71 & Durango & 141 & 0.90 \\
\hline Veracruz & 1024 & 6.51 & Baja California Norte & 127 & 0.81 \\
\hline Morelos & 803 & 5.10 & San Luis Potosí & 117 & 0.74 \\
\hline Guanajuato & 585 & 3.72 & Colima & 113 & 0.72 \\
\hline Nuevo León & 522 & 3.32 & Sinaloa & 94 & 0.60 \\
\hline Yucatán & 474 & 3.01 & Oaxaca & 90 & 0.57 \\
\hline Chiapas & 456 & 2.90 & Tabasco & 85 & 0.54 \\
\hline Jalisco & 431 & 2.74 & Aguascalientes & 73 & 0.46 \\
\hline Sonora & 404 & 2.57 & Zacatecas & 69 & 0.44 \\
\hline Coahuila & 334 & 2.12 & Nayarit & 62 & 0.39 \\
\hline Baja California Sur & 304 & 1.93 & Quintana Roo & 55 & 0.35 \\
\hline Michoacán & 263 & 1.67 & Guerrero & 53 & 0.34 \\
\hline Tamaulipas & 256 & 1.63 & Tlaxcala & 43 & 0.27 \\
\hline Puebla & 202 & 1.28 & Campeche & 22 & 0.14 \\
\hline \multirow[t]{2}{*}{ Querétaro } & 202 & 1.28 & Hidalgo & 17 & 0.11 \\
\hline & & & $\begin{array}{l}\text { Total de artículos } \\
\text { analizados } \dagger\end{array}$ & 15736 & \\
\hline
\end{tabular}

$\dagger$ El número total de artículos (15 736) es el de la base de datos, y no la suma de los artículos por Estado (16 009), porque contabiliza los artículos publicados en colaboración y en los que pueden participar dos o más estados. 


\section{Producción científica por sectores institucionales}

El sector en el que se publicaron más artículos es el que agrupa a las Universidades, con casi $57 \%$ de participación en la producción total. El peso de la investigación de este sector recae fundamentalmente en las universidades públicas $(55.59 \%)$ mientras que las privadas sólo publican $1.21 \%$. En segundo lugar se encuentran los institutos o centros de investigación y los investigadores de estos dos sectores en conjunto participaron con $92.05 \%$ del total, en contraste con los científicos de instituciones internacionales $(5.73 \%)$. Los investigadores del resto de tipos de instituciones participaron con $3.59 \%$ de los artículos (Cuadro 2).

Cuadro 2. Producción de artículos de revistas científicas en ciencias agrícolas, por tipo de institución (1983-2002).

\begin{tabular}{lcr}
\hline Instituciones & $\begin{array}{c}\text { Núm. de } \\
\text { artículos }\end{array}$ & $\%$ \\
\hline Universidades & 8939 & 56.80 \\
Institutos o centros de investigación & 5548 & 35.25 \\
Instituciones internacionales & 902 & 5.73 \\
Hospitales & 159 & 1.01 \\
Gobierno & 151 & 0.95 \\
Industrias & 116 & 0.73 \\
Otro tipo & 143 & 0.90 \\
Total de artículos en la base de datos & $\mathbf{1 5 7 3 6}$ & \\
\hline
\end{tabular}

La producción de los investigadores de las universidades se mantuvo al alza durante todo el periodo, aunque con algunos altibajos; la tasa más alta fue en 2000 (63.54 \%) (Figura 2). Aunque en valores absolutos la producción científica de los institutos de investigación creció, los porcentajes decrecieron de $39.39 \%$ en 1983 a $33.05 \%$ en 2002 , con una tasa media en la primera mitad del periodo de $41.46 \%$ y de $31.1 \%$ en la segunda mitad. La tasa de participación en la publicación de artículos de las instituciones internacionales tuvo un crecimiento negativo, con altibajos en el periodo ya que fue de $6.97 \%$ en 1983 a $4.11 \%$ en 2002. La tasa de producción de los investigadores de las instituciones de gobierno mostró una fuerte caída, de $6.06 \%$ en 1983 a $0.67 \%$ en 2002; en cambio, la de los científicos de los hospitales creció, ya que en 1983 su tasa fue de $0.91 \%$ y en 2002 de $1.51 \%$. También los investigadores de las instituciones clasificadas como "industrias" duplicaron su producción científica, pues en 1983 participaron con $0.30 \%$ y en 2002 subió a $0.67 \%$ (Figura 2).

Estos resultados ratifican el preponderante papel que tienen las universidades, concretamente las públicas, y los institutos de investigación en la producción científica de las ciencias agrícolas en México, papel que aumentó en los últimos años del estudio. Al respecto, Russell (1995) encontró que alrededor de $70 \%$ de la investigación científica se realizó en universidades públicas. En los informes de CONACYT $(1997,2003)$, aunque no mencionan la tasa de participación de las universidades se infiere que es la más alta ya que las primeras cuatro instituciones más productivas son universidades públicas. Quizá la principal razón por las que las universidades lideran la producción científica en México se deba a que son las promotoras, las protagonistas y las principales consumidoras del esfuerzo en investigación científica en los países subdesarrollados (Galina y Russell, 1994).

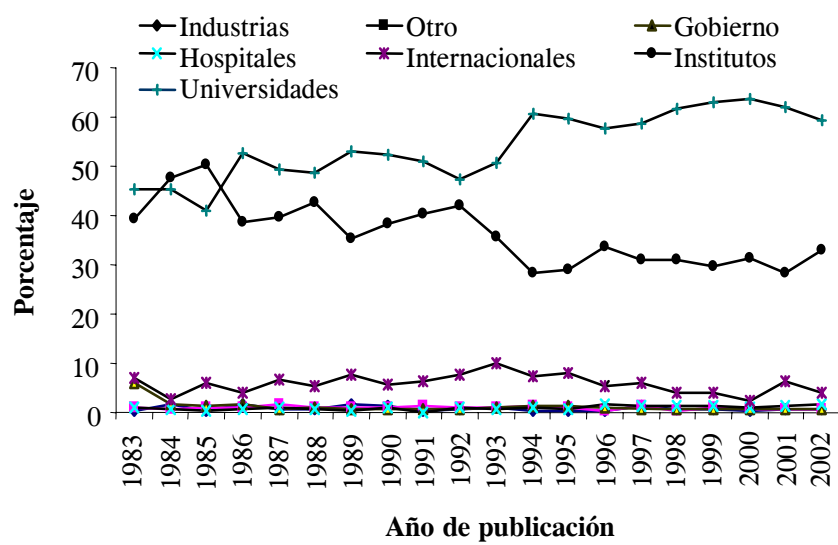

Figura 2. Evolución porcentual anual de la producción de artículos publicados en revistas, por tipo de institución (1983-2002).

\section{Instituciones a las que pertenecen los autores}

En este apartado se analizan solamente las instituciones cuyos investigadores participan con al menos $1 \%$ del total de artículos publicados durante el periodo de estudio. Destaca la Universidad Nacional Autónoma de México como la institución que alberga a los investigadores que publican más artículos en ciencias agrícolas en México, con una tasa de $25.19 \%$. Siguen a esta universidad los científicos de instituciones dedicadas por entero a la investigación en ciencias agrícolas, como el Instituto Nacional de Investigaciones Forestales Agrícolas y Pecuarias y el Colegio de Postgraduados en Ciencias Agrícolas, así como la Universidad Autónoma Chapingo. Estas cuatro instituciones en conjunto publicaron más de $50 \%$ de los artículos (Cuadro 3).

Los investigadores de las siguientes instituciones: Centro Internacional de Mejoramiento de Maíz y Trigo (CIMMYT), Centro de Investigación y Estudios Avanzados, Instituto de Ecología en Veracruz, Universidad Autónoma de Nuevo León, Universidad Autónoma Metropolitana, Centro de Investigaciones Biológicas del Noreste, Escuela Nacional de Biología, Universidad de Guadalajara, Universidad Autónoma de Yucatán, Colegio de la Frontera Sur y la Universidad Autónoma Agraria Antonio Narro, en conjunto contribuyeron con $26.08 \%$ de los artículos publicados. De las anteriores 15 instituciones, 
solamente el CIMMYT no es universidad pública o centro de investigación, ya que está clasificado como institución internacional (Cuadro 3).

Cuadro 3. Instituciones mexicanas o asentadas en México a la que pertenecen los autores de artículos de revistas en ciencias agrícolas (1983-2002).

\begin{tabular}{|c|c|c|}
\hline Instituciones & $\begin{array}{l}\text { Núm. de } \\
\text { artículos }\end{array}$ & $\%$ \\
\hline Universidad Nacional Autónoma de México & 3964 & 25.19 \\
\hline $\begin{array}{l}\text { Instituto Nacional de Investigaciones } \\
\text { Agrícolas y Pecuarias }\end{array}$ & 1874 & 11.91 \\
\hline $\begin{array}{l}\text { Colegio de Postgraduados en Ciencias } \\
\text { Agrícolas }\end{array}$ & 1608 & 10.22 \\
\hline Universidad Autónoma Chapingo & 945 & 6.01 \\
\hline $\begin{array}{l}\text { Centro Internacional de Mejoramiento de } \\
\text { Maíz y Trigo }\end{array}$ & 770 & 4.89 \\
\hline $\begin{array}{l}\text { Centro de Investigaciones y Estudios } \\
\text { Avanzados del IPN }\end{array}$ & 697 & 4.43 \\
\hline Instituto de Ecología en Veracruz & 583 & 3.70 \\
\hline Universidad Autónoma de Nuevo León & 421 & 2.68 \\
\hline Universidad Autónoma Metropolitana & 391 & 2.48 \\
\hline $\begin{array}{l}\text { Centro de Investigaciones Biológicas del } \\
\text { Noreste }\end{array}$ & 268 & 1.70 \\
\hline $\begin{array}{l}\text { Escuela Nacional de Ciencias Biológicas } \\
\text { del IPN }\end{array}$ & 222 & 1.41 \\
\hline Universidad de Guadalajara & 213 & 1.35 \\
\hline Universidad Autónoma de Yucatán & 190 & 1.21 \\
\hline El Colegio de la Frontera Sur & 180 & 1.14 \\
\hline $\begin{array}{l}\text { Universidad Autónoma Agraria Antonio } \\
\text { Narro }\end{array}$ & 172 & 1.09 \\
\hline 343 Instituciones & 3636 & 23.11 \\
\hline Total de artículos & 15736 & \\
\hline
\end{tabular}

La Universidad Nacional Autónoma de México contribuye con la tasa más alta de producción de artículos en ciencias agrícolas sin tener una facultad de agricultura, pues cuenta con facultades e institutos que hacen investigación en todas las áreas de la ciencia en diferentes regiones de la República Mexicana (Delgado y Russell, 1992). Estas altas tasas no son sorpresivas, ya que esta institución es la que más contribuye a la ciencia mexicana (CONACYT, 1997, 2003, 2004). En México, las universidades privadas generalmente no se dedican a la investigación científica; su tarea fundamental es la formación de recursos humanos, aunque hay excepciones como el Instituto Tecnológico y de Estudios Superiores de Monterrey que no aparece en el Cuadro 3 por tener un porcentaje de publicación de $0.53 \%$, inferior al $1 \%$ considerado.

Entre las instituciones gubernamentales destacaron la Secretaría de Agricultura, Ganadería y Desarrollo Rural, actualmente denominada Secretaría de Agricultura, Ganadería, Desarrollo Rural, Pesca y Alimentación; la Comisión Nacional del Agua; y la Comisión Nacional para el Conocimiento y Uso de la Biodiversidad, con porcentajes de publicación de $0.57,0.06$ y $0.02 \%$ respectivamente. Entre las industrias destacaron Campbell's Sinalopasta y
Apicultura Cardoso, pero como en los casos anteriores su porcentaje de publicaciones fue muy bajo $(0.04 \%$ en ambas industrias).

\section{Idiomas en los que se publicaron los artículos}

El idioma en el que más se publicaron artículos fue el inglés (62.33\%), seguido del español (37.9\%). En estos dos idiomas se publicó casi la totalidad de artículos, lo que muestra el alto grado de uso del idioma inglés en la publicación de la investigación que se realiza en las ciencias agrícolas, pero también pone de manifiesto el gran interés que se tiene en mantener al español para la difusión de los resultados (Cuadro 4).

Cuadro 4. Valores absolutos y porcentuales de los idiomas en los que se publicaron artículos de revistas en ciencias agrícolas (1983-2002).

\begin{tabular}{lcr}
\hline Idioma & Núm. de artículos & $\%$ \\
\hline Inglés & 9808 & 62.33 \\
Español & 5963 & 37.90 \\
Francés & 103 & 0.66 \\
Alemán & 31 & 0.19 \\
Portugués & 17 & 0.11 \\
Italiano & 4 & 0.03 \\
Ruso & 3 & 0.02 \\
Árabe & 2 & 0.01 \\
Chino & 1 & 0.01 \\
Danés & 1 & 0.01 \\
Japonés & 1 & 0.01 \\
Vietnamita & 1 & 0.01 \\
Total de artículos & $\mathbf{1 5 3 6}$ & $\mathbf{1 0 0 . 0 0}$ \\
\hline
\end{tabular}

\section{Revistas que concentran la mayoría artículos}

En este caso el análisis se limitó a identificar a las revistas donde se publicó la mitad del total de artículos, y encontró que en 57 revistas (3.64\%) se publicaron 7867 artículos (50.05\%); el resto se publicó en 1506 títulos diferentes $(96.35 \%)$, de los cuales $624(39.98 \%)$ publicaron únicamente un artículo cada uno, o sea $3.96 \%$ de artículos. De las 57 revistas que publicaron la mitad de los artículos, 21 de ellas se editan en México y en conjunto publicaron 5968 artículos (37.92\%); 22 revistas se publican en los Estados Unidos de América con 1758 artículos $(11.17 \%)$; tres se editan en el Reino Unido, donde se publicaron 365 artículos (2.32\%); tres revistas se editan en Alemania, en las cuales se publicaron 189 artículos (1.2\%); ocho revistas se publican en Argentina, Holanda, Italia, Irlanda, Costa Rica y Guatemala, y publicaron 612 artículos (3.89\%) (Cuadro 5).

De las 13 revistas en las que se publicó el mayor número de artículos, diez se editan en México: Agrociencia, Revista Chapingo, Veterinaria México, Técnica Pecuaria en México, Folia Entomológica Mexicana, Revista Mexi- 
cana de Fitopatología, Terra, Ciencia Forestal, Agricultura Técnica en México y Revista Fitotecnia Mexicana. Las cinco principales revistas editadas en otros países y en las que se publicó un número importante de artículos son: Phytochemistry, Crop Science, Phyton, Southwestern Entomologist y Journal of Natural Products (Cuadro 5). De las revistas que publicaron $50 \%$ de los artículos, $36.84 \%$ de ellas se editan en México, lo que demuestra el gran interés que tienen las revistas nacionales para difundir este tipo de investigación de aplicación para el entorno social que la sufraga. El conocer las revistas que más artículos publican sobre una determinada temática ayuda a los científicos en la tarea de seleccionar aquéllas en las cuales puedan publicar los resultados de investigación. Facilita además la tarea de seleccionar las revistas nacionales e internacionales y de corriente principal en las bibliotecas y centros de documentación.

Cuadro 5. Títulos de revistas en las que se publicó $44 \%$ de los artículos hechos por investigadores que laboran en alguna institución mexicana (1983-2002).

\begin{tabular}{|c|c|c|c|c|}
\hline Revistas & País de publicación & Núm. de artículos & $\%$ & $\%$ Acumulado \\
\hline Agrociencia & México & 1173 & 7.45 & 7.45 \\
\hline Revista Chapingo & México & 707 & 4.49 & 11.95 \\
\hline Veterinaria México & México & 675 & 4.29 & 16.24 \\
\hline Técnica Pecuaria en México & México & 395 & 2.51 & 18.75 \\
\hline Folia Entomológica Mexicana & México & 307 & 1.95 & 20.70 \\
\hline Revista Mexicana de Fitopatología & México & 291 & 1.85 & 22.55 \\
\hline Phytochemistry & Reino Unido & 233 & 1.48 & 24.03 \\
\hline Crop Science & EE. UU. & 219 & 1.39 & 25.42 \\
\hline Terra & México & 209 & 1.33 & 26.75 \\
\hline Ciencia Forestal & México & 180 & 1.14 & 27.89 \\
\hline Agricultura Técnica en México & México & 178 & 1.13 & 29.02 \\
\hline Phyton & Argentina & 159 & 1.01 & 30.03 \\
\hline Revista Fitotecnia Mexicana & México & 146 & 0.93 & 30.96 \\
\hline Southwestern Entomologist & EE. UU. & 131 & 0.83 & 31.79 \\
\hline Journal of Natural Products & EE. UU. & 104 & 0.66 & 32.45 \\
\hline Acta Botánica Mexicana & México & 101 & 0.64 & 33.10 \\
\hline Revista Latinoamericana de Microbiología & México & 100 & 0.64 & 33.73 \\
\hline Journal of Agricultural and Food Chemistry & EE. UU. & 94 & 0.60 & 34.33 \\
\hline Mycotaxon & EE. UU. & 90 & 0.57 & 34.90 \\
\hline Journal of Food Science & EE. UU. & 88 & 0.56 & 35.46 \\
\hline Journal of Ethnopharmacology & Irlanda & 79 & 0.50 & 35.96 \\
\hline Biotropica & EE. UU. & 78 & 0.50 & 36.46 \\
\hline Plant Disease & EE. UU. & 78 & 0.50 & 36.95 \\
\hline Small Ruminant Research & EE.UU. & 77 & 0.49 & 37.44 \\
\hline Journal of Arid Environments & Reino Unido & 76 & 0.48 & 37.93 \\
\hline Journal of Bacteriology & EE. UU. & 76 & 0.48 & 38.41 \\
\hline Archivos Latinoamericanos de Nutrición & Guatemala & 72 & 0.45 & 38.87 \\
\hline Bean Improvement Cooperative & EE. UU. & 72 & 0.46 & 39.32 \\
\hline Theoretical and Applied Genetics & Alemania & 72 & 0.46 & 39.78 \\
\hline American Journal of Botany & EE. UU. & 71 & 0.45 & 40.23 \\
\hline Annals of the Entomological Society of America & EE. UU. & 71 & 0.45 & 40.68 \\
\hline Journal of Economic Entomology & EE. UU. & 71 & 0.45 & 41.14 \\
\hline Florida Entomologist & EE. UU. & 70 & 0.45 & 41.58 \\
\hline Turrialba & Costa Rica & 70 & 0.45 & 42.03 \\
\hline Planta Médica & Alemania & 69 & 0.44 & 42.46 \\
\hline Revista Mexicana de Micología & México & 67 & 0.43 & 42.89 \\
\hline Agronomía Mesoamericana & México & 65 & 0.41 & 43.30 \\
\hline Madera y Bosques & México & 65 & 0.41 & 43.72 \\
\hline Euphytica & Holanda & 64 & 0.41 & 44.12 \\
\hline 18 revistas para completar $50 \%$ & ---------- & 934 & 5.94 & 50.06 \\
\hline 1506 revistas el restante $50 \%$ & ----------- & 7859 & 49.94 & 100 \\
\hline
\end{tabular}

Revistas que publicaron $50 \%$ de artículos: 57; Núm. de artículos que conforman $50 \%$ : 7877. 
En los años subsecuentes al periodo de estudio, como se puede constatar en los informes anuales del estado de la ciencia en México del CONACYT, se observa un aumento de la producción científica en revistas de alto impacto, especialmente con la inclusión en el ISI Web of Science de Agrociencia y Revista Fitotecnia Mexicana, ambas ya con factor de impacto (Thomson Reuters, 2007) y más recientemente en las revistas Acta Botánica Mexicana, Boletín de la Sociedad Botánica de México, Hidrobiológica, Revista Internacional de Contaminación Ambiental y Revista Mexicana de Biodiversidad.

\section{Colaboración científica entre autores}

Debido a las limitaciones de las bases de datos Agricola, Cab Abstracts, Agris y Tropag \& Rural, no se calcularon otros tipos de colaboración (entre instituciones o entre países). La disminución de artículos firmados por un solo autor y el aumento de los firmados por dos o más autores dieron un comportamiento simétrico con valores de correlación y determinación altos $\left(r=0.96\right.$ y $-0.96, R^{2}=$ 0.93). Este comportamiento muestra la tendencia a publicar en colaboración. La tasa media de artículos firmados por un solo autor para todo el periodo de estudio fue $12.38 \%$, y $87.62 \%$ para los artículos firmados por dos o más autores (Figura 3).

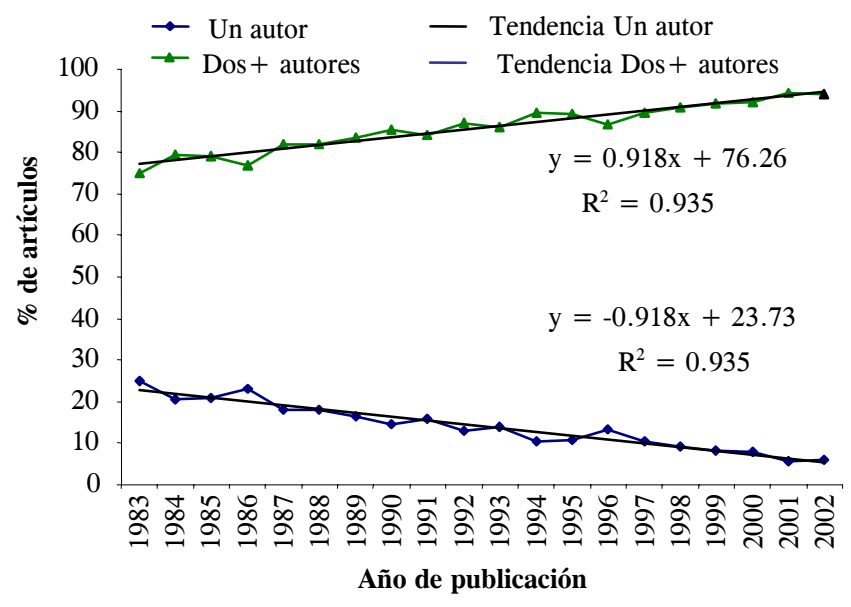

Figura 3. Tasas anuales y tendencia de crecimiento de artículos en ciencias agrícolas, con y sin colaboración de autores (1983-2002).

Según Prpic citado por Sanz y Martín (1997), la tasa de artículos firmados por más de un autor es de casi $80 \%$ para ciencias puras y experimentales, frente a la de ciencias sociales y humanidades que es de $80 \%$ para artículos firmados por un solo autor. En este trabajo, la tasa de artículos firmados por más de un autor fue $87.6 \%$. El índice de coautoría de 1983 fueron 2.47 autores por artículo, que aumentó a 4.08 para el año 2002, con valores de $\mathrm{r}$ $=0.95, R^{2}=0.90$ (Figura 4).

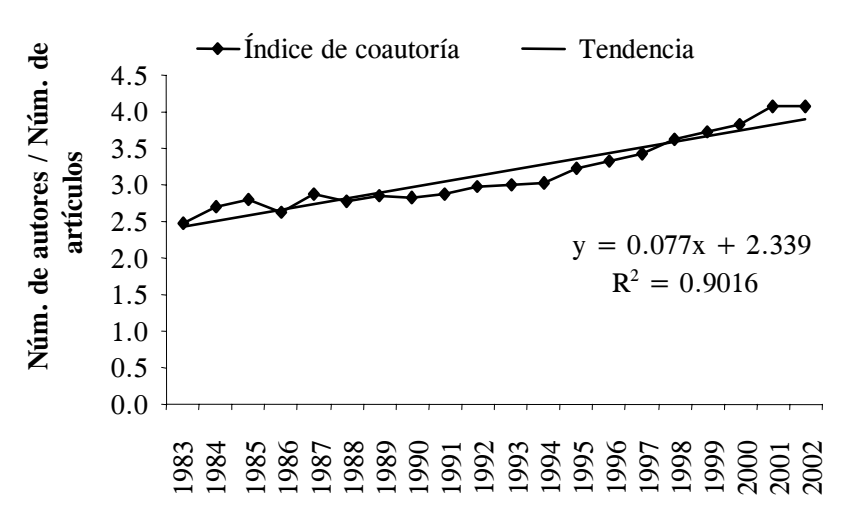

Año de publicación

Figura 4. Índice de coautoría anual y tendencia de crecimiento del índice (1983-2002).

La media del índice de coautoría en la primera mitad del periodo (1983-1992) fue de 2.81, y en la segunda mitad (1993-2002) fue de 3.56. Esto indica que los autores tienden a publicar más en conjunto en los años recientes, lo que fortalece los grupos de investigación. Estos índices son congruentes con lo reportado por Sancho (1990), quien afirmó que el índice varía entre disciplinas pero que para ciencias se puede considerar entre 2.5 y 3.5. Según López y Terrada (1992), a principios del siglo XX $80 \%$ de los trabajos provenían de autores únicos, mientras que a finales de ese siglo en $80 \%$ de los trabajos había entre 3 y 3.5 autores. Sin embargo, se debe tener cuidado al interpretar este índice de coautoría, pues Sanz y Martín (1997) hacen notar que puede estar afectado por factores ilegítimos, como la necesidad de mejorar el currículo de los integrantes del grupo de investigación para acceder a becas y financiamiento de investigaciones, lo que conduce a autorías no justificadas.

\section{CONCLUSIONES}

La producción científica mexicana en ciencias agrícolas aumentó significativamente de 1983 a 2002, sobre todo en los últimos diez años de dicho periodo cuando casi se triplicó lo publicado en los primeros diez. La producción científica se concentró en el Distrito Federal y el Estado de México, las regiones de mayor desarrollo y donde tienen su sede muchas instituciones de educación e investigación en el área. La mayoría de dicha producción fue realizada por universidades y centros públicos de investigación.

Las instituciones internacionales participaron con un pequeño porcentaje en la producción científica en el área, y los hospitales, agencias gubernamentales e industria produjeron un porcentaje aún menor. 
En conjunto, la Universidad Nacional Autónoma de México, el Instituto Nacional de Investigaciones Forestales, Agrícolas y Pecuarias, el Colegio de Postgraduados y la Universidad Autónoma Chapingo participaron en la publicación de más de $50 \%$ de los artículos.

Los investigadores mexicanos o asentados en México publicaron la mayoría de sus artículos en inglés y en español. Del total de revistas que publicaron la mitad de los artículos, $36.84 \%$ se editan en México y conjuntaron $37.92 \%$ de los artículos. Se observó una disminución de artículos firmados por un solo autor, y el índice de coautoría aumentó de 2.47 autores por artículo en 1983 a 4.08 en 2002.

\section{BIBLIOGRAFÍA}

Alfaraz P H, A M Calviño (2004) Bibliometric study on food science and technology: scientific production in Iberian-American countries (1991-2000). Scientometrics 61:89-102.

Anta E, J A Rivera, C S Galina, A Porras, L Zarco J M Russell (1989) Análisis de la información publicada en México sobre eficiencia reproductiva de los bovinos: I. Estudio bibliométrico. Vet. Méx. 20:3-10.

Aréchiga U, H (1995) La Investigación Científica y Tecnológica. Asociación Nacional de Universidades e Instituciones de Educación Superior. México. 93 p.

Arenas M, M P Dovalina, J Licea de A (2004) La investigación agrícola en América Latina y el Caribe desde una perspectiva bibliométrica. An. Docum. 7:29-38.

CONACYT (1997) Indicadores de Actividades Científicas y Tecnológicas: México. Consejo Nacional de Ciencia y Tecnología, CONACYT. México. 220 p.

CONACYT (2003) Informe General del Estado de la Ciencia y la Tecnología: México. Consejo Nacional de Ciencia y Tecnología, CONACYT. México. $238 \mathrm{p}$.

CONACYT (2004) Informe General del Estado de la Ciencia y la Tecnología: México. Consejo Nacional de Ciencia y Tecnología, CONACYT. México. 384 p.

D'alessandro E, P Cárdenas, J M Russell, C S Galina (2000) La revista Veterinaria México como medio de difusión de la investigación en ciencias veterinarias y zootécnicas. Vet. Méx. 31:261-266.

Delgado H, J M Russell (1992) Impact of studies published in the international literature by scientists at the National University of Mexico. Scientometrics 23:75-90.

Ekboir J, J A Espinosa G, J J Arellano E, G Moctezuma L, A Tapia N (2003) Análisis del Sistema Mexicano de Investigación Agropecuaria. Centro Internacional de Mejoramiento de Maíz y Trigo. México. $33 \mathrm{p}$.

Gaillard J, J M Russell, A Furó T, N Narvaez B, E Zink (2001) IFS Impact in Mexico: 25 Years of Support to Scientists. International Foundation for Science. Stockholm. 151 p.
Galina C S, J M Russell (1994) Transfer of research findings in the tropics; how are researchers transferring information to livestock producers? World Anim. Rev. 80/81:3-12.

Galina C S, P Riverol, M Cárdenas, M Aguilar, J M Russell (2000) The impact of the International Foundation for Science (IFS) funding on Latin American research in animal health and reproduction. Interciencia 25:30-35.

González U L (1997) Teoría de la ciencia, documentación y bibliometría. Rev. Gral. Inf. Docum. 7:2001-215.

Licea de A J, M Sandoval, A Arenas (2003) La investigación agrícola en México, con enfoque de género. An. Docum. 6:145-154.

Lomnitz L A, L Mayer (1994) Veterinary medicine and animal husbandry in Mexico: from empiricism to science and technology. Minerva 32:144-157.

López P J M, M L Terrada (1992) Los indicadores bibliométricos y la evaluación de la actividad medico-científica: (III). Los indicadores de producción, circulación y dispersión, consumo de la información y repercusión. Med. Clín. (Barcelona) 98:142148 .

Mirande A, J M Russell, C S, Galina, R Navarro-Fierro (1987) Research in animal reproduction and analysis of the contribution made by Latin America. Theriogenology 28:121-127.

Nasir A M, H K Asan, A Hamid, S S Agha (1994) Bibliometric evaluation of agricultural literature published in Malaysia. Scientometrics 29:191-217.

Russell J M (1995) The increasing role of international cooperation in science and technological research in Mexico. Scientometrics 34:45-61.

Russell J M, M S Correa N, N García A, J A Guadarrama H, L Priego O (1987) Research and publication trends of a Latin American veterinary faculty. Interciencia 12:243-244.

Russell J M, C S Galina (1987) Research and publishing trends in cattle reproduction in the tropics: Part 2. A third world prerogative. Anim. Breeding Abstr. 55:819-828.

Russell J M, C S Galina (1988). Productivity of authors publishing on tropical bovine reproduction. Interciencia 13:311-313.

Russell J M, C S Galina, E Anta, A Porras, L Zarco (1990) Bibliographical studies concerning of cattle in the tropics. In: Livestock Reproduction in Latin America: Research Coordination Meeting (Bogotá: 1988): Proceedings. International Atomic Energy Agency, Vienna. pp:285-295.

Russell J M, M Mendoza, G Martínez (1987) Patterns of literature citation by undergraduate students and researchers in the veterinary field. Scientometrics 12:73-80.

Saavedra F O, G Sotolongo A, M V Guzmán S (2002) Medición de la producción científica en América Latina y el Caribe en el campo agrícola y afines: un estudio bibliométrico. Rev. Esp. Docum. Cient. 25:151-161.

Sancho R (1990) Indicadores bibliométricos utilizados en la evaluación de la ciencia y la tecnología. Rev. Esp. Docum. Cient. 13:842-865

Sanz C E, C Martín M (1997) Técnicas bibliométricas aplicadas a los estudios de usuarios. Rev. Gral. Inf. Docum. 7:41-68.

Spinak E (1996) Diccionario Enciclopédico de Bibliometría, Cienciometría e Informetría. UNESCO. Caracas. 343 p.

Thomson Reuters (2007) Scientific Journal of Citation Reports. Disponible en http://scientific.thomsonreuters.com (Julio 18, 2008). 